\title{
The Hamilton Apportionment Method Is Between the Adams Method and the Jefferson Method
}

\author{
Luc Lauwers \\ Center for Economic Studies, K. U. Leuven, Naamsestraat 69, 3000 Leuven, Belgium, \\ luc.lauwers@econ.kuleuven.be, http://www.econ.kuleuven.be/Luc.Lauwers/public \\ Tom Van Puyenbroeck \\ European University College Brussels (EHSAL), Stormstraat 2, 1000 Brussels, Belgium, \\ Center for Economic Studies, K. U. Leuven, Naamsestraat 69, 3000 Leuven, Belgium, tom.vanpuyenbroeck@ehsal.be
}

\begin{abstract}
The Adams apportionment method is the only divisor method that consistently favors small districts relative to the Hamilton method. The Jefferson method is the only divisor method that favors large districts relative to the Hamilton method. These statements hold for the Balinski and Young [3] interpretation of "favoring small/large districts," and for the partial "majorization ranking" introduced by Marshall et al. [7]. As such, Hamilton is positioned in between Adams and Jefferson.
\end{abstract}

Key words: apportionment; proportional representation; discrete allocation; divisor methods; majorization MSC2000 subject classification: Primary: 90B80, 91B12; secondary: 91B14, 91F10

OR/MS subject classification: Primary: government; secondary: elections

History: Received January 12, 2005; revised August 18, 2005.

1. Introduction. Apportionment methods are used to round the population proportions of electoral districts (or the vote proportions of parties) to integer numbers of seats in a representative body. The translation of almost continuous proportions into natural numbers nearly always involves adjusting. As the gain or loss of a single seat is usually considered important, the effect of the rounding process is a central issue in the theory of apportionment. In particular, different apportionment methods are tested on whether or not they systematically favor the smaller districts over the larger districts (or the larger over the smaller). There are at least two ways to study this phenomenon. First, one can study the seat bias, either by estimates from empirical data or by exact formulae derived from a probability model. Second, one can try to rank different methods according to the preferential treatment of smaller versus larger districts. Before we recall some of the literature on both tracks, we illustrate the following apportionment methods:

- Hamilton: a quota method with residual fit by the largest remainders

- Adams: the divisor method with rounding up

- Jefferson: the divisor method with rounding down

- Webster: the divisor method with standard rounding

These methods are properly defined below. For now, it suffices to see how they allocate 10 seats over the three-tuple $(603,249,148)$. The total population amounts to 1,000 . We list the outcomes in Table 1. Moving in this table from the left to the right, the small districts 2 and 3 each hand in one seat to the large district. The focus is on whether this example exposes fundamental properties of the apportionment methods.

Balinski and Young [3, Appendix A, §5] use historical data to calculate the average percentage bias of some divisor methods. They observe that (i) the method of Adams favors the smaller districts the most; (ii) the Webster method is close to unbiased; and (iii) the Jefferson method has a plain bias toward the larger districts. Recently, Schuster et al. [8], Schwingenschlögl and Drton [9], and Drton and Schwingenschlögl [5] confirm and extend these results on the basis of exact formulae for the expected bias. With respect to the Hamilton method, Schuster et al. [8] expose that its seat bias is practically zero.

Concerning the second track in the literature, Balinski and Young [3, p. 118] formalize the idea of favoring small districts as follows. Method $M^{\prime}$ favors small districts relative to method $M$ if moving from an $M^{\prime}$-allocation toward an $M$-allocation; it cannot happen that simultaneously a smaller district loses seats and a larger district gains seats. In view of the results on seat bias, one might expect that Adams favors small districts relative to Webster and Jefferson, and that Webster favors small districts relative to Jefferson. As a matter of fact, these results appear in Balinski and Young [3, Proposition 5.1, p. 119].

Marshall et al. [7] observe that, in general, the Balinski-Young relation fails transitivity. They propose a weaker (and transitive) relation as follows. Consider an apportionment problem. First, order the districts from large to small. Denote a seat allocation proposed by Adams by $\left(a_{1}, a_{2}, \ldots, a_{s}\right)$ and an allocation proposed by Jefferson by $\left(b_{1}, b_{2}, \ldots, b_{s}\right)$. Then, they show that the following holds:

$$
a_{1} \leq b_{1}, \quad a_{1}+a_{2} \leq b_{1}+b_{2}, \ldots \quad \text { and } \quad a_{1}+a_{2}+\cdots+a_{s-1} \leq b_{1}+b_{2}+\cdots+b_{s-1},
$$


TABLE 1

\begin{tabular}{lccccc}
\hline District & Proportions & Adams & Webster & Hamilton & Jefferson \\
\hline 1. 603 & 6.03 & 5 & 6 & 6 & 7, \\
2. 249 & 2.49 & 3 & 3 & 3 & 2, \\
3. 148 & 1.48 & 2 & 1 & 1 & 1, \\
$+1,000$ & 10.00 & 10 & 10 & 10 & 10. \\
\hline
\end{tabular}

where $s$ is the number of districts. The totals $a_{1}+a_{2}+\cdots+a_{s}$ and $b_{1}+b_{2}+\cdots+b_{s}$, of course, coincide. Marshall et al. [7] thus show that the Adams method is majorized by the Jefferson method. They use this majorization criterion to rank a number of divisor methods. The relationships between Adams, Webster, Jefferson, and other divisor methods obtained by Balinski and Young are recovered.'

Because the Hamilton apportionment method is not a divisor method, the above results are all silent about the positioning of the Hamilton method (and other quota methods) in these rankings. Still, the Hamilton method was and is today widely used. This paper attempts to fill the gap. Our first result reads:

Proposition 1.1. (i) The method of Adams favors small districts relative to the method of Hamilton. (ii) The method of Hamilton favors small districts relative to the method of Jefferson.

Hence, in terms of majorization we obtain that Adams is majorized by Hamilton, and that Hamilton is majorized by Jefferson.

Finally, we explore the (im)possibilities of refining the above proposition. Apparently, some quota methods are not in between the methods of Adams and Jefferson. Furthermore, we illustrate the incompleteness of the majorization relation: The Hamilton method is incomparable to other divisor methods such as Dean, Hill, and Webster. This final observation generalizes to the following characterization within the class of divisor methods.

THEOREM 1.1. (i) There is only one divisor method that favors small districts relative to the Hamilton apportionment method. It is the method of Adams. (ii) There is only one divisor method that favors large districts relative to the Hamilton apportionment method. It is the method of Jefferson.

The next section introduces the notation and provides the definitions. In $\S 3$, we prove Proposition 1.1. Section 4 characterizes the methods of Adams and Jefferson within the class of divisor methods.

2. Notations and definitions. A specific problem of apportionment is an $s$-tuple $p=\left(p_{1}, p_{2}, \ldots, p_{s}\right)$ of populations and an integer size of house $h$. Without loss of generality, $p$ is ordered from large to small: $p_{1} \geq$ $p_{2} \geq \cdots \geq p_{s}>0$. Let $\mathscr{P}^{s}$ collect all apportionment problems $(p, h)$, with $p$ an ordered $s$-tuple of positive natural numbers and $h$ a positive natural number. Let $\mathbb{N}=\{0,1,2, \ldots\}$ collect the natural numbers. An apportionment method $M$ is a correspondence

$$
M: \mathscr{P}^{s} \longrightarrow \mathbb{N}^{s}:(p, h) \longmapsto M(p, h),
$$

enforcing the condition $a_{1}+a_{2}+\cdots+a_{s}=h$ for each $a$ in $M(p, h)$.

An ideal allocation is the $s$-tuple $q(p, h)$ of quota defined by

$$
q(p, h)=\left(q_{1}, q_{2}, \ldots, q_{s}\right), \quad \text { with } q_{k}=\frac{p_{k}}{p_{1}+p_{2}+\cdots+p_{s}} \times h .
$$

Because the quota vector usually is outside the set $\mathbb{N}^{s}$, the next requirement seems natural.

An apportionment method $M$ is said to respect the lower quota (upper quota) if for each apportionment problem $(p, h)$, for each $a$ in $M(p, h)$, and for each $i=1,2, \ldots, s$ we have

$$
\left\lfloor q_{i}\right\rfloor \leq a_{i} \quad\left(a_{i} \leq\left\lceil q_{i}\right\rceil\right),
$$

where the floor $\lfloor r\rfloor$ (resp. the ceiling $\lceil r\rceil$ ) of a positive real number $r$ is the largest natural number smaller (resp. the smallest natural number greater) than or equal to $r$. In case $r$ belongs to $\mathbb{N}$, then $\lfloor r\rfloor=\lceil r\rceil=r$.

The Hamilton method or the method of the largest remainders is the prototype example of a method that respects the lower and upper quotas. Let us recall its definition. Let $(p, h)$ be an apportionment problem. Calculate the quota $q(p, h)$ and allocate $\left\lfloor q_{i}\right\rfloor$ seats to each district $i$. As such, a total of $\left\lfloor q_{1}\right\rfloor+\left\lfloor q_{2}\right\rfloor+\cdots+\left\lfloor q_{s}\right\rfloor$

\footnotetext{
'As a matter of fact, when restricted to the class of divisor methods, the Balinski and Young relation and the "is majorized by" relation coincide (Lauwers and Van Puyenbroeck [6]).
} 
seats is allocated. Give the remaining $\sum_{i}\left(q_{i}-\left\lfloor q_{i}\right\rfloor\right)$ seats one each to districts having the largest remainders $q_{i}-\left\lfloor q_{i}\right\rfloor$.

Whereas quota methods respect the lower and upper quotas by construction, divisor methods do not. ${ }^{2}$ In fact, within the class of divisor methods, the methods of Jefferson and Adams are the only ones that respect one of the quota (Balinski and Young [3, Proposition 6.4, p. 130]). The method of Jefferson is defined as follows: Start by rewriting the population $s$-tuple $p$ as

$$
p^{1}=\left(\frac{p_{1}}{1}, \frac{p_{2}}{1}, \ldots, \frac{p_{s}}{1}\right)
$$

Allocate the first seat to the district with the highest $p^{1}$-coordinate (by assumption, this is District 1 ). Then the vector $p^{1}$ is replaced by the vector

$$
p^{2}=\left(\frac{p_{1}}{2}, \frac{p_{2}}{1}, \ldots, \frac{p_{s}}{1}\right)
$$

The district with the highest $p^{2}$-coordinate wins the second seat. The vector $p^{2}$ transforms to the vector $p^{3}$ by increasing the denominator of the winning district with one. Repetition of this algorithm generates a sequence $p^{1}, p^{2}, p^{3}, \ldots$ After $h$ steps, the total number of seats is allocated to the districts. A tie in the final quotients generates multiple apportionments. Jefferson's method is the only divisor method that stays above the lower quota. The method of Adams proceeds similarly. The only difference is that this method starts from the vector

$$
\tilde{p}^{1}=\left(\frac{p_{1}}{0}, \frac{p_{2}}{0}, \ldots, \frac{p_{s}}{0}\right)
$$

and assumes that $p_{i} / 0>p_{j} / 0>r$ whenever $p_{i}>p_{j}$ and whatever the value of $r$ in $\mathbb{R}$. Each time a district has the largest value, it wins an additional seat and the corresponding denominator increases with one: The vector $\tilde{p}^{t}$ transforms into $\tilde{p}^{t+1}$. Again, after $h$ steps the procedure ends. In case $h \geq s$ the method of Adams is obtained by (i) allocating one seat to each district and (ii) applying the method of Jefferson on $\tilde{p}^{s+1}=p^{1}$ to allocate the remaining $h-s$ seats. The method of Adams stays below the upper quota.

Observe that the method of Jefferson uses the sequence $1,2,3, \ldots$ as subsequent denominators for each district, whereas Adams uses $0,1,2, \ldots$ In general, one can define a method for each strictly increasing divisor sequence. As such, the sequence $0.5,1.5,2.5, \ldots$ defines the method of Webster.

All methods discussed in this paper satisfy weak monotonicity (Balinski and Young [3, p. 147]): If district $i$ is smaller than district $j$ (i.e., if $p_{i}<p_{j}$ ), then each of these methods allocates at least as many seats to district $j$ as it does to district $i$. Now, let us formalize the concepts of favoring small districts and of majorization.

Definition 2.1. Let $M$ and $M^{\prime}$ be two apportionment methods. Then $M^{\prime}$ favors small districts relative to $M$ if for each apportionment problem $(p, h)$ in $\mathscr{P}^{s}$, for each $a$ in $M(p, h)$, and for each $a^{\prime}$ in $M^{\prime}(p, h)$ we have

$$
p_{i}<p_{j} \text { implies either } a_{i}^{\prime} \geq a_{i} \text { or } a_{j}^{\prime} \leq a_{j} .
$$

We visualize this relationship through Tables 1-3. We indicate a seat transfer when moving from a first toward a second allocation by an arrow that starts from the losing district in the first allocation and arrives at the winning district in the second allocation. If $M^{\prime}$ favors small districts relative to $M$, then each arrow has a positive slope (we order the districts from large to small, and we put the $M^{\prime}$-column in front of the $M$-column).

Definition 2.2. Let $M$ and $M^{\prime}$ be two weakly monotone apportionment methods. Then $M^{\prime}$ is majorized by $M$ if for each apportionment problem $(p, h)$ in $\mathscr{P}^{s}$ with $p_{1}>p_{2}>\cdots>p_{s}$, for each $a$ in $M(p, h)$, for each $a^{\prime}$ in $M^{\prime}(p, h)$, and for each $k=1,2, \ldots, s-1$ we have

$$
a_{1}^{\prime}+a_{2}^{\prime}+\cdots+a_{k}^{\prime} \leq a_{1}+a_{2}+\cdots+a_{k} .
$$

In words, method $M^{\prime}$ is majorized by $M$ if method $M$ always allocates a higher cumulative total number of seats to the larger districts than $M^{\prime}$ does. In Definition 2, equal population sizes are excluded. A tie $p_{i}=p_{i+1}$ may disturb the relationship. For example, the Adams and the Jefferson methods propose two apportionments to the problem $(p ; h)=(10,10 ; 3)$, to wit, $(2,1)$ and $(1,2)$. Only the allocation $(2,1)$ appears in decreasing order.

As already mentioned, the notion "is majorized by" is less demanding than "favoring small districts relative to" (Marshall et al. [7, Lemma]).

\footnotetext{
${ }^{2}$ On the other hand, quota methods are prone to the Alabama paradox, whereas divisor methods are not. Balinski and Young [2] reconcile both desiderata in the quotatone methods. We are not aware of any practical implementation of these methods.
} 
TABLE 2. A $>$ Hamilton $>J$, but not Hamilton $>D, H, W$.

\begin{tabular}{ccccc}
\hline Population & Proportions & $A, D, H, W$ & Hamilton & $J$ \\
\hline 603 & 6.70 & 6 & 7 & 8 , \\
149 & 1.66 & 2 & 2 & 1 , \\
148 & 1.64 & 2 & 1 & 1 , \\
+900 & 10.00 & 10 & 10 & 10. \\
\hline
\end{tabular}

TABLE 3. A Hamilton $>J$, but not $D, H, W>$ Hamilton.

\begin{tabular}{rcccc}
\hline Population & Proportions & $A$ & Hamilton & $D, H, W, J$ \\
\hline 1,600 & 5.36 & 5 & 5 & 6, \\
1,005 & 3.37 & 3 & 4 & 3, \\
380 & 1.27 & 2 & 1 & 1, \\
$+2,985$ & 10.00 & 10 & 10 & 10. \\
\hline
\end{tabular}

3. Hamilton lies in between Adams and Jefferson. We first compare the methods of Adams and Hamilton. Consider a problem $(p, h)$ in $\mathscr{P}^{s}$. For each district $i$,

$q_{i}$ denotes the quota $p_{i} h /\left(p_{1}+p_{2}+\cdots+p_{s}\right)$,

$l_{i}$ denotes the lower quota $\left\lfloor q_{i}\right\rfloor$,

$z_{i}$ denotes the decimal part of the quota $\left(q_{i}=l_{i}+z_{i}\right)$,

$c$ denotes the Hamilton apportionment with $c_{1} \geq c_{2} \geq \cdots \geq c_{s}$ (with $l_{i} \leq c_{i} \leq\left\lceil q_{i}\right\rceil$ ), and

$a$ denotes the Adams apportionment with $a_{1} \geq a_{2} \geq \cdots \geq a_{s}$ (recall $\left.a_{i} \leq\left\lceil q_{i}\right\rceil\right)$.

Note that in case of a population tie $\left(p_{i}=p_{i+1}\right)$, we select the particular solution that favors those districts that appear earlier in the list $\left(c_{i} \geq c_{i+1}\right.$ and $\left.a_{i} \geq a_{i+1}\right)$. This convention has no consequences for the main results. Its purpose is to simplify the next lemma in which the transition from $c=\left(c_{1}, c_{2}, \ldots, c_{s}\right)$ toward $a=\left(a_{1}, a_{2}, \ldots, a_{s}\right)$ is investigated. When moving from $c$ to $a$, a district might lose $\left(c_{i}>a_{i}\right)$ or win $\left(c_{i}<a_{i}\right)$ one or more seats, or the district might experience a status quo $\left(c_{i}=a_{i}\right)$.

Lemma 3.1. Consider the move from $c$ (Hamilton) to a (Adams). Collect the winners in $W$ and the losers in $L$. Then, for each $j$ in $L$ and $i$ in $W$ we have $j<i$. Furthermore, the cardinality of $W$ is less than or equal to $\left(l_{1}+l_{2}+\cdots+l_{s}\right)+s-h$.

Proof. The Adams apportionment $a$ is determined through the following equilibrium inequality (Balinski and Young [1]):

$$
\max \left\{\frac{p_{i}}{a_{i}} \mid a_{i} \geq 0\right\} \leq \min \left\{\frac{p_{i}}{a_{i}-1} \mid a_{i}>0\right\} .
$$

We use the linear homogeneity of the maximum and the minimum rule to rewrite this inequality (multiply each number by $\left.h /\left(p_{1}+p_{2}+\cdots+p_{s}\right)\right)$ :

$$
\max \left\{\frac{l_{i}+z_{i}}{a_{i}} \mid a_{i} \geq 0\right\} \leq \min \left\{\frac{l_{i}+z_{i}}{a_{i}-1} \mid a_{i}>0\right\}
$$

In case the $s$-tuple $c$ also satisfies the above inequality (substituting $a_{i}$ for $c_{i}$ ), then the Hamilton outcome $c$ and the Adams outcome $a$ coincide. Therefore, let us assume that $c$ does not satisfy (1).

Let $l_{1}+l_{2}+\cdots+l_{s}=h-k$. Thus, Hamilton allocates an extra seat (additional to the lower quota) to $k$ districts. Let $Z_{1}$ collect those $k$ districts $i$ for which $c_{i}=l_{i}+1$. The remaining districts obtain their lower quota according to the Hamilton method. Let $Z_{0}$ collect these $s-k$ districts.

Because the method of Adams respects the upper quota, it holds that moving from $c$ to $a$, only those districts that belong to $Z_{0}$ can receive one extra seat. Therefore, moving from $c$ to $a$, the maximum number of winners is bounded by $s-k .^{3}$

Hence, $W \subseteq Z_{0}$, and the number of winners is bounded by $s-k$. The cardinality of the set $L$ is less than or equal to the cardinality of $W:$ A district in $L$ may hand in more than one seat. Assume that according to

\footnotetext{
${ }^{3}$ In the problem $(p, h)=(96,1,1,1,1 ; 5)$ this upper bound of $s-k=4$ transfers is reached.
} 
Adams's method, loser $j$ has to hand in $r_{j} \geq 1$ seats, i.e., $a_{j}=c_{j}-r_{j}$. Calculate the fractions $\left(l_{j}+z_{j}\right) /\left(c_{j}-r_{j}\right)$ for the losing districts and $\left(l_{i}+z_{i}\right) / l_{i}$ for the winning districts. Then the following inequality must hold:

$$
\max \left\{\frac{l_{j}+z_{j}}{c_{j}-r_{j}} \mid j \text { is a loser }\right\} \leq \min \left\{\frac{l_{i}+z_{i}}{l_{i}} \mid i \text { is a winner }\right\},
$$

still with the assumption that $x_{1}>x_{2}>x_{3}>0$ implies $x_{2} / 0>x_{3} / 0>x_{1}$. Indeed, should the reverse strict inequality hold, then according to the method of Adams at least one loser would end up with at least one seat too many. Let $j$ be a losing and $i$ a winning district. Because of the particular selections $a$ and $c$, it cannot happen that $p_{i}=p_{j}$. The previous inequality implies

$$
\frac{l_{i}+z_{i}}{l_{i}}=1+\frac{z_{i}}{l_{i}} \geq \frac{l_{j}+z_{j}}{c_{j}-r_{j}}
$$

District $j$ either belongs to $Z_{0}$ or to $Z_{1}$. In the first case, we have $c_{j}=l_{j}$, and the inequality simplifies to

$$
\frac{l_{i}+z_{i}}{l_{i}}=1+\frac{z_{i}}{l_{i}} \geq \frac{l_{j}+z_{j}}{l_{j}-r_{j}}=1+\frac{r_{j}+z_{j}}{l_{j}-r_{j}} \quad \text { or } \quad \frac{l_{j}-r_{j}}{l_{i}} \geq \frac{r_{j}+z_{j}}{z_{i}}>1 .
$$

It follows that $l_{j}>l_{i}+r_{j} \geq l_{i}+1$. As a consequence, we obtain $j<i$.

In the second case $\left(j\right.$ in $\left.Z_{1}\right)$, we have $h_{j}=l_{j}+1$, and we obtain

$$
1+\frac{z_{i}}{l_{i}} \geq \frac{l_{j}+z_{j}}{l_{j}+1-r_{j}}=1+\frac{r_{j}-1+z_{j}}{l_{j}+1-r_{j}} \quad \text { or } \quad \frac{l_{j}+1-r_{j}}{l_{i}} \geq \frac{r_{j}-1+z_{j}}{z_{i}} \geq 1 .
$$

The final inequality follows from the fact that either $r_{j}>1$ or $r_{j}=1$ and $z_{j} \geq z_{i}$ (the Hamilton method allocates extra seats to those districts having a higher decimal part in the quota). We end up with $l_{j} \geq l_{i}+r_{j}-1 \geq l_{i}$. It follows that $j<i$.

Conclude in both cases that the winning district $i$ is smaller than the losing district $j$, i.e., $j<i$ (remember that $p_{1} \geq p_{2} \geq \cdots \geq p_{s}$ ).

Next, we focus on the comparison of Hamilton and Jefferson. We stay in the same vocabulary and in the same notation. Let $b=\left(b_{1}, b_{2}, \ldots, b_{s}\right)$ denote the apportionment proposed by Jefferson: Again we select the particular solution that in a population tie favors the districts that appear earlier in the list. The analogue of Lemma 3.1 holds.

Lemma 3.2. Consider the move from $c$ (Hamilton) to $b$ (Jefferson). Collect the winners in $W$ and the losers in $L$. Then, for each $j$ in $L$ and $i$ in $W$ we have $j>i$. Furthermore, the cardinality of $L$ is less than or equal to $h-\left(l_{1}+l_{2}+\cdots+l_{s}\right)$.

Proof. The proof follows the same steps as the previous one. Hence, we concentrate on the differences. The equilibrium inequality for Jefferson's method reads (after multiplying by $h / \sum p_{i}$ )

$$
\max \left\{\frac{l_{1}+z_{1}}{b_{1}+1}, \frac{l_{2}+z_{2}}{b_{2}+1}, \ldots, \frac{l_{s}+z_{s}}{b_{s}+1}\right\} \leq \min \left\{\frac{l_{1}+z_{1}}{b_{1}}, \frac{l_{2}+z_{2}}{b_{2}}, \ldots, \frac{l_{s}+z_{s}}{b_{s}}\right\} .
$$

The method of Jefferson respects the lower quota. Hence, when moving from the Hamilton outcome $c$ to the Jefferson outcome $b$, only those districts that belong to $Z_{1}$ can hand in one seat. Therefore, the upperbound for the number of losers is equal to $k$.

Let $j$ be a losing and $i$ a winning district. Assume that according to Jefferson, the winning district $i$ gains $r_{i} \geq 1$ seats, i.e., $b_{i}=c_{i}+r_{i}$. Then, it must be the case that

$$
\frac{l_{j}+z_{j}}{l_{j}+1}=1-\frac{1-z_{j}}{l_{j}+1} \leq \frac{l_{i}+z_{i}}{c_{i}+r_{i}}
$$

Indeed, should the reverse strict inequality hold, then district $j$ with $l_{j}$ seats would have a higher priority than district $i$ with $c_{i}+r_{i}-1$ seats and would obtain (according to Jefferson) a next seat.

District $i$ either belongs to $Z_{0}$ or to $Z_{1}$. In the first case, we have $c_{i}=l_{i}$, and the previous inequality simplifies to

$$
\frac{l_{j}+z_{j}}{l_{j}+1}=1-\frac{1-z_{j}}{l_{j}} \leq \frac{l_{i}+z_{i}}{l_{i}+r_{j}}=1-\frac{r_{i}-z_{i}}{l_{i}+r_{i}} \quad \text { or } \quad \frac{l_{i}+r_{i}}{l_{j}+1} \geq \frac{r_{i}-z_{i}}{1-z_{j}} \geq 1 .
$$


It follows that $b_{i}=l_{i}+r_{i} \geq l_{j}+1=b_{j}+1$. As a consequence, we obtain $i<j$.

In the second case ( $i$ in $Z_{l}$ ), we have $h_{i}=l_{i}+1$. Therefore,

$$
1-\frac{1-z_{j}}{l_{j}+1} \leq \frac{l_{i}+z_{i}}{l_{i}+1-r_{j}}=1-\frac{1+r_{i}-z_{i}}{l_{i}+1+r_{i}} \quad \text { or } \quad \frac{l_{i}+1+r_{i}}{l_{j}+1} \geq \frac{1+r_{i}-z_{i}}{1-z_{j}}>1 .
$$

We end up with $b_{i}=l_{i}+1+r_{i}>l_{j}+1=b_{j}+1$. It follows that $i<j$.

We conclude that in both cases the winning district $i$ is larger than the losing district $j$, i.e., $i<j$.

Now, we give a proof of Proposition 1.1(i): Adams's method favors small districts relative to Hamilton. The proof of the second statement (ii) is similar.

Proof of Proposition 1.1(i). Consider two districts $i$ and $j$ such that $p_{i}<p_{j}$. Let us move from an Hamilton allocation $c$ toward an Adams allocation $a$, and let us compare $c_{i}$ with $a_{i}$ and $c_{j}$ with $a_{j}$. From Lemma 3.1 we know that in the transition from $c$ to $a$ the combination of $i$ losing and $j$ winning, i.e., $c_{i}>a_{i}$ and $c_{j}<a_{j}$, does not occur. Therefore, the statement $a_{i} \geq c_{i}$ or $a_{j} \leq c_{j}$ holds.

Corollary 3.1. (i) Adams's method is majorized by Hamilton. (ii) Hamilton's method is majorized by Jefferson.

Proof. This is a consequence of Proposition 1.1 in combination with the lemma of Marshall et al. [7].

In view of these results, we investigate whether or not the methods of Adams and Jefferson embrace all quota methods. In other words, does it hold that Adams's method is majorized by $Q$ and that $Q$ is majorized by Jefferson as soon as the method $Q$ respects the lower and the upper quotas?

The answer is negative. A counterexample is found in the strongest list method. (Broquet and Lancelot [4] include this method in the list of actually used apportionment methods.) This method first allocates the lower quota and then gives the remaining seats one each to the largest districts. Application of this method to the apportionment problem $(p ; h)=(11,10,9,6,4 ; 29)$ results in $(8,8,7,4,2)$. Jefferson leads to $(8,7,7,4,3)$. Hence, in this example Jefferson gives the second-largest district one seat less.

Analogously, the counterpart of the strongest list method is incomparable to Adams. Indeed, consider the method $Q^{*}$ that first allocates the lower quota and then gives the remaining seats one each to the smallest districts. (Taking into account the weak monotonicity constraint: If adding one seat to district $i+1$ conflicts with the number of seats of district $i$, then one should first add one seat to $i$.) This method respects both quota and the weak monotonicity axiom. Nevertheless, for some problems this method allocates more seats to small districts than Adams does: $Q^{*}$ applied to the problem $(28,10,9,6,4 ; 29)$ leads to $(14,5,4,3,3)$ where Adams proposes $(14,5,5,3,2)$.

In conclusion, satisfying the lower and upper quotas does not guarantee a positioning in between the methods of Adams and Jefferson.

4. Characterizations in the class of divisor methods. Up to now, Hamilton is positioned in between the methods of Adams and Jefferson. The next question we tackle is whether this result can be refined by introducing other popular divisor methods such as Webster, Dean, and Hill. In order to define these methods, it suffices to present the divisor sequence (as explained in $\S 2$ ):

(A) Adams, sequence $d(0)=0, d(1)=1, d(2)=2, \ldots$, or $d(a)=a$,

$(J)$ Jefferson, sequence $d(0)=1, d(1)=2, d(2)=3, \ldots$, or $d(a)=a+1$,

(W) Webster, $d(a)=a+0.5$,

(D) Dean, $d(a)=a(a+1) /(a+0.5)$,

(H) Hill, $d(a)=\sqrt{a(a+1)}$.

Each divisor sequence $d(a)$ defined for each $a$ in $\mathbb{N}$ and satisfying $a \leq d(a) \leq a+1$ is called a divisor method (Balinski and Young [3, p. 99]). Balinski and Young [3, p. 119] prove $A>D>H>W>J$, with ">" a shorthand for "favors small states relative to." The same inequalities reappear in Marshall et al. [7, p. 894]. As such, it is natural to check where the Hamilton method fits in this ranking.

Apparently, the statement $A>$ Hamilton $>J$ cannot be narrowed down. Let us illustrate this claim by means of two examples: see Table 2 and Table 3.

Inspired by these examples, we investigate the existence of divisor methods that favor small districts relative to Hamilton. Theorem 1.1 indicates that only the method of Adams owns this property.

Proof of Theorem 1.1(i). From Proposition 1.1, we know that the method of Adams favors small districts relative to Hamilton. Let us show that Adams is the only divisor method with this property: For each divisor sequence $d(a)$ different from the divisor sequence of Adams, we construct an apportionment problem for which Hamilton allocates more seats to the smallest district. 
Let the divisor sequence $d(a)$ be defined for each $a$ in $\mathbb{N}$ and satisfying $a \leq d(a) \leq a+1$. Recall that the divisor method of Adams is defined through the sequence $d^{*}(a)=a$. Assume that the sequence $d(a)$ differs from $d^{*}(a)$. Let $a^{*}$ be the smallest natural number for which $d\left(a^{*}\right) \neq a^{*}$. Let $d\left(a^{*}\right)=a^{*}+\varepsilon$ with $0<\varepsilon \leq 1$. Consider an apportionment problem with $s$ districts $(\varepsilon>1 / s)$ and with the following quota:

$$
q_{1}=q_{2}=\cdots=q_{s-1}=l+z>q_{s}=a^{*}+z_{s},
$$

where the decimal parts $z$ and $z_{s}$ satisfy $\varepsilon>z_{s}>z>0$ and $(s-1) z+z_{s}=1$. The size of the house is equal to $h=(s-1) l+\left(a^{*}+1\right)$. Hence, the Hamilton allocation $c$ reads $c_{1}=c_{2}=\cdots=c_{s-1}=l$ and $c_{s}=a^{*}+1>0$.

Manipulating the value of $l$, we will force the divisor method to allocate less than $a^{*}+1$ seats to the smallest district $s$. We want the following inequality to hold:

$$
\frac{a^{*}+z_{s}}{a^{*}+\varepsilon}<\frac{l+z}{d(l)}
$$

In other words, we want that according to the divisor sequence $d(a)$ a large district has a priority over the small district to obtain an $(l+1)$ th seat. The construction implies

$$
\frac{a^{*}+z_{s}}{a^{*}+\varepsilon}<1 \quad \text { and } \quad \frac{l+z}{l+1} \leq \frac{l+z}{d(l)} .
$$

Take $l$ sufficiently large and obtain.

$$
\frac{a^{*}+z_{s}}{a^{*}+\varepsilon}<\frac{l+z}{l+1} \leq \frac{l+z}{d(l)}
$$

As a consequence, the divisor method $d(a)$ allocates fewer seats to the smallest district than Hamilton does.

Analogously, the method of Jefferson is the only divisor method that favors "large" districts relative to Hamilton.

ProOF OF TheOREM 1.1(ii). From Proposition 1.1, we know that Hamilton favors small districts relative to Jefferson. Let us show that Jefferson is the only divisor method with this property: We construct for each divisor sequence $d(a)$ different from the divisor sequence of Jefferson, an apportionment problem for which Hamilton allocates fewer seats to the smallest district.

Let the divisor sequence $d(a)$ be defined for each $a$ in $\mathbb{N}$ and satisfying $a \leq d(a) \leq a+1$. Recall that Jefferson uses the sequence $d^{*}(a)=a+1$. Assume that the sequence $d(a)$ differs from $d^{*}(a)$ and let $a^{*}$ be the smallest natural number such that $d\left(a^{*}\right) \neq a^{*}+1$. Let $d\left(a^{*}\right)=a^{*}+\varepsilon$ with $0 \leq \varepsilon<1$. Consider an apportionment problem with $s$ districts $(1-\varepsilon>1 / s)$ and with the following quota:

$$
q_{1}=q_{2}=\cdots=q_{s-1}=l+z>q_{s}=a^{*}+z_{s},
$$

where the decimal parts $z$ and $z_{s}$ satisfy $1>z>z_{s}>\varepsilon$ and $(s-1) z+z_{s}=s-1$. Let the size of the house be equal to $h=(s-1)(l+1)+a^{*}$. Hamilton allocates $l+1$ seats to each large district and $a^{*}$ seats to the small district. Manipulating the value of $l$ we will force the divisor method to allocate more than $a^{*}$ seats to the smallest district $s$ :

$$
\frac{a^{*}+z_{s}}{a^{*}+\varepsilon}>\frac{l+z}{d(l)}
$$

This can be done because

$$
\frac{a^{*}+z_{s}}{a^{*}+\varepsilon}>1 \quad \text { and } \quad \frac{l+z}{l} \geq \frac{l+z}{d(l)} .
$$

Indeed, as $l$ goes to infinity the fraction $(l+z) / l$ converges to 1 . Hence, for $l$ sufficiently large, the divisor method $d(a)$ allocates at least $a^{*}+1$ seats to the smallest district.

The proofs indicate that both statements in Theorem 1.1 also hold when "favoring small states relative to" is replaced with the relation "is majorized by."

Acknowledgments. We thank the three anonymous referees for insightful comments that have improved the content and the presentation of the paper.

\section{References}

[1] Balinski, M. L., H. P. Young. 1978. The Jefferson method of apportionment. SIAM Rev. 20(2) 278-284. 
[2] Balinski, M. L., H. P. Young. 1979. Quotatone apportionment methods. Math. Oper. Res. 4(1) 31-38.

[3] Balinski, M. L., H. P. Young. 1982. Fair Representation: Meeting the Ideal of One Man, One Vote. Yale University Press, New Haven, CT, London, UK.

[4] Broquet, Ch., A. Lancelot. 2004. Report on electoral systems: Overview of available solutions and selection criteria (adopted by the Venice Commission at its 57th Plenary Session). Study 250/003, Council of Europe, http://www.venice.coe.int/docs/2004/CDLAD(2004)003-e.pdf.

[5] Drton, M., U. Schwingenschlögl. 2005. Asymptotic seat bias formulas. Metrika 62(1) 23-31.

[6] Lauwers, L., T. Van Puyenbroeck. 2005. The Balinski-Young comparison of divisor methods is transitive. Social Choice Welfare. http://dx.doi.org/10.1007/s00355-006-01 10-6.

[7] Marshall, A. W., I. Olkin, F. Pukelsheim. 2002. A majorization comparison of apportionment methods in proportional representation. Social Choice Welfare 19(4) 885-900.

[8] Schuster, K., F. Pukelsheim, M. Drton, N. R. Draper. 2003. Seat bias apportionment methods for proportional representation. Electoral Stud. 22(4) 651-676.

[9] Schwingenschlögl, U., M. Drton. 2004. Seat allocation distributions and seat biases of stationary apportionment methods for proportional representation. Metrika 60(2) 191-202. 
Copyright 2006, by INFORMS, all rights reserved. Copyright of Mathematics of Operations Research is the property of INFORMS: Institute for Operations Research and its content may not be copied or emailed to multiple sites or posted to a listserv without the copyright holder's express written permission. However, users may print, download, or email articles for individual use. 